\title{
L'âgisme, un grave problème social: que peut-on faire à ce sujet?
}

L'article que signent dans ce numéro Chipperfield et Havens met en lumière toute la question de l'âgisme. L'âgisme, qui est la somme de toutes ces attitudes négatives, basées sur des stéréotypes et discriminatoires à l'encontre des personnes âgées, constitue un problème important et complexe, aussi bien en regard du vieillissement que de la société en général. Ce problème est important dans la mesure où il affecte de façon significative les personnes âgées d'aujourd'hui comme celles de demain. De plus, sa difficulté est liée au fait que l'âgisme se manifeste souvent de façon subtile.

Comme le mettent bien en évidence Chipperfield et Havens, il y a lieu de faire un lien entre la perception du respect qui nous est manifesté et le respect de soi en tant que tel. Certes, il est vrai qu'un certain nombre de personnes âgées ne seront pas vraiment influencées par les comportements et attitudes âgistes d'autrui. Par contre, pour un grand nombre d'entre elles, ces comportements et attitudes peuvent être une source de diminution de l'estime d'elles-mêmes et peuvent conduire à une diminution de bien-être et même de qualité de vie.

On peut rechercher l'origine du problème de l'âgisme dans le fait que les êtres humains ne sont pas coupés les uns des autres. Nous nous trouvons tous dans un monde en compagnie d'autres personnes. En d'autres termes, être un humain, c'est être en interaction. Nous choisissons ce que nous allons penser de nous-mêmes, mais ces choix s'opèrent dans un contexte partiellement personnel et partiellement social. Ainsi, toute l'imagerie ou les concepts que nous élaborons face à notre propre vieillissement (Kennen, Birren, \& Schroots, 1991) sont influencés par d'autres concepts concernant le vieillissement et qui émanent, eux, d'une structure sociale extérieure.

Et c'est à un point tel que l'âgisme devient un sujet crucial. Cela est dû au fait que beaucoup de personnes âgées intègrent ces images négatives du vieillissement qui leur sont imposées de l'extérieur, en dépit du fait que leur perception personnelle ne correspond pas nécessairement aux images renvoyées par les autres. Comme le mentionne Philibert (1968, p. 302) les personnes âgées peuvent fort bien se rendre compte que ces images "âgistes " sont contestables: "Certes elles les croient provisoires et révocables mais elles doivent les accepter pour vivre et pour agir ». Souvent, beaucoup de personnes âgées se résignent face aux attitudes âgistes, mais le prix à payer peut être fort élevé, aussi bien individuellement qu'en terme de perte de la contribution sociale potentielle que pourrait apporter un secteur important de notre population.

Il est intéressant de remarquer que les attitudes âgistes n'affectent pas seulement des personnes de soixante-cinq ans et plus. Dans le milieu du tra- 
vail, par exemple, on peut subir beaucoup plus tôt des discriminations fondées sur l'âge. C'est un fait reconnu que beaucoup de personnes recherchant un emploi à l'âge de quarante ans rencontrent de sérieuses difficultés. Ces difficultés sont souvent dues aux interactions avec des employeurs plus jeunes qui manifestent des comportements âgistes et de plus, se trouvent très ambivalents par rapport à leur propre vieillissement.

Nous pouvons nous tourner vers la médecine pour découvrir une autre source d'âgisme, très significative. Beaucoup d'attitudes négatives et des postulats tout à fait inexacts, concernant, entre autres, les capacités des gens et leur niveau de santé, émanent couramment des médecins (Davidson, 1991; Lebel, 1991). À la source de ce problème se trouve l'âgisme qui peut être perçu comme un ensemble de comportements négatifs irréfléchis et de concepts implicites relatifs à l'âge, tout à fait dommageables.

Cette idée de concept implicite met en lumière d'un côté, la subtilité des comportements âgistes et, de l'autre, les difficultés inhérentes aux efforts menés pour combattre l'âgisme. Les discriminations à l'encontre des personnes âgées (et de celles qui ne le sont pas encore) ne procèdent pas, le plus souvent, d'une attitude réfléchie. Ainsi se veut la nature de toute discrimination: il y a, au départ, un automatisme auquel s'ajoute en effet d'entraînement. Cela signifie que l'âgisme commence par être un stéréotype dans l'esprit des individus ou des groupes, peu importe la raison, pour devenir ensuite très explicite, sous la forme de politiques sociales acceptant ou excluant des personnes ayant un âge chronologique donné, apparent ou réel. Dans ce contexte, l'âgisme s'exprime sous la forme du caractère obligatoire de la retraite et de la prestation de services de soins de santé, fondée sur l'âge des bénéficiaires.

Tout cela constitue des exemples de conditions extérieures négatives qui facilitent l'intégration, par les personnes âgées, de perceptions personnelles restrictives ou carrément négatives. Ces situations fondées sur l'âgisme marginalisent les personnes âgées et l'objectif de suivre l'itinéraire de vie qu'elles ont choisi devient alors plus difficile pour elles. Il est évident qu'un souci de dialogue et de recherche sur les problèmes de l'âgisme devient nécessaire, aux niveaux individuel et sociétal, dans une société civilisée qui se dit fondée sur des valeurs humaines et sur des principes d'éthique.

Jusqu'à présent, nous avons mentionné que l'âgisme constitue un sujet complexe et même crucial, tant pour les individus que pour la société et en particulier dans une société qui vieillit rapidement comme au Canada. Toutefois, il n'est pas suffisant de signaler le problème, ni même de l'analyser; il est également nécessaire de discuter de solutions possibles. À ce sujet, on peut distinguer trois principes généraux susceptibles de nous aider à contrecarrer l'âgisme.

En premier lieu, les connaissances actuelles devraient nous amener à être très prudents avant de souscrire à des affirmations à connotation âgiste concernant la fin de la vie, à l'effet, par exemple, que « toutes les personnes âgées sont des ceci ou des cela " ou bien que " toutes les personnes âgées déclinent ou encore continuent à progresser ". Le problème principal lié à 
ce type d'affirmations généralisantes ne réside pas dans l'affirmation que le déclin ou le progrès existe, mais dans cette sorte de disjonction qui donnerait à cette période de fin de la vie une fonction exclusive de progrès ou de déclin. Quand ces affirmations se voient décerner un statut officiel, elles conduisent souvent à la tentative bien intentionnée, mais tout à fait erronée, de s'assurer que chacun se conforme à un ensemble de caractéristiques ou encore à un modèle particulier. Ce qui est requis ici, c'est une évaluation critique des connaissances de base utilisées pour guider des décisions d'intervention. Un tel dessein sera facilité aussi bien par une large ouverture sur les orientations multiples et les dimensions liées à l'étude du vieillissement, que par une bonne appréciation de la nature complexe et interdisciplinaire de ces connaissances de base. En d'autres termes, comme le dirait Aristote, nous devons nous rapprocher de l'individu. Une éducation à tous les niveaux, formel et informels, et des politiques pour supporter une telle éducation constituent un instrument efficace, mais encore peu développé, pour combattre l'âgisme.

Cependant, les différents types d'éducation possibles ne suffisent pas en soi. La seconde directive est la suivante: les praticiens, les chercheurs et, au sens large du terme, tous ceux qui font partie de cette société vieillissante doivent prêter attention à leurs propres perceptions et conclusions face au vieillissement. Nous devons développer des approches nouvelles pour rendre explicites nos perceptions implicites. On a besoin de programmes et d'interventions qui créent des situations auxquelles seraient exposés des praticiens et des décideurs et qui entreraient en interaction avec les personnes âgées. C'est ainsi que les professionnels et les autres pourront renouveler et améliorer leurs perceptions sur le vieillissement (Birren, 1987), ce qui veut dire qu'ils percevront le vieillissement et les personnes âgées d'une façon plus appropriée. Mais, encore plus important, ils cesseront de projeter leurs propres perceptions pour prendre conscience de celles des personnes âgées. Ils comprendront toute la signification du vieillissement comme de l'intérieur d'une autre personne. C'est une chose de posséder une connaissance plus pointue du vieillissement et c'en est une autre d'agir et de mettre ses connaissances en pratique, en interagissant avec les personnes âgées. Seules la connaissance et une action appropriée peuvent contrer l'âgisme. D'ailleurs, le second point est plus difficile à réaliser. Il est difficile parce qu'il faut beaucoup de temps et d'effort pour changer nos perceptions professionnelles et personnelles. En plus, nous faison face au problème discuté plus haut, à l'effet qu'il nous faut tenir compte de cette ambivalence vécue par de nombreux intervenants, quand il s'agit de parler de leur propre vieillissement et du sens de la vieillesse. En conséquence, nous nous devons d'avoir le désir ou l'intention d'entamer ce dialogue.

La troisième directive pour contrer l'âgisme consiste à se centrer sur la personne âgée en tant que personne. Cela signifie qu'on peut accomplir beaucoup en s'écartant de la spécificité de l'âge comme tel et en mettant l'accent sur ce que telle personne âgée en particulier ne peut pas faire et, plus important encore, sur ce qu'elle peut faire (Davidson, 1991). Cela vaut pour 
tous les contextes comme la médecine, l'éducation, le travail et la famille. Certes, suggérer qu'il est important de traiter les personnes âgées comme des personnes est plus facile à dire qu'à faire. Tenter de comprendre une personne âgée comme un individu, en bonne ou en mauvaise santé et doté d'un passé, d'un présent et d'un futur uniques, demande davantage d'efforts et de temps.

Les directives que nous suggérons ici font pression sur le système et sur les ressources disponibles dans ce système. Cependant, notre connaissance du vieillissement nous indique que nous devenons de plus en plus individualistes avec l'âge et que des interactions avec les personnes âgées, sur la base de positions âgistes ou généralisantes, sont inappropriées. Dans les faits, l'âgisme est insoutenable à la fois parce qu'il privilégie une image universelle du vieillissement et parce que cette image universelle est négative. Par exemple, l'âgisme exclut toute considération de thèmes comme le sens de la personne et la spiritualité perçus comme parties intégrantes de la personne humaine. À ce sujet, nous pouvons nous poser la question du sens de vieillir dans une société âgiste. En plus de ce que signifie pour nous la connaissance du vieillissement, nous nous retrouvons ici face à un impératif d'ordre éthique. L'éthique nous dit, en effet, que toute personne doit être traitée comme un être autonome, doté de droits fondamentaux. En conséquence, cela veut dire être une personne, quel que soit son âge, sa santé, son revenu, etc. ...

Deux points demeurent finalement à l'ordre du jour. En premier lieu, les praticiens, en tant que personnes impliquées directement dans l'existence des personnes âgées, occupent une position privilégiée pour contrecarrer l'âgisme et ses effets négatifs. Le praticien ou l'intervenant jouent des rôles essentiels en transmettant des perceptions d'espoir, de dignité et de sens de la vie à des personnes qui se trouvent souvent dans des positions vulnérables. Bien plus, ceux qui agissent ainsi trouvent qu'il s'agit là d'une expérience extrêmement enrichissante pour toutes les personnes concernées.

Le second et dernier point apporte une certaine réserve aux vues optimistes exprimées plus haut. Le passage d'une société âgiste à une société où l'âge n'entre pas en question (Neugarten \& Hagestad, 1976), à une société qui développerait plus de flexibilité envers les perceptions personnelles et sociales du vieillissement, et où les personnes âgées disposeraient de plus de choix, suppose une collaboration de tous les responsables des politiques sociales et institutionnelles. Une société post-industrielle comme le Canada comporte beaucoup d'éléments de gestion sociale de la vie humaine. Individuellement, les personnes âgées peuvent tellement faire pour contrer l'âgisme et on peut dire la même chose des praticiens. Dans un société fondée sur l'âgisme, il existe des contraintes structurelles qui requièrent des efforts à tous les paliers de gouvernement. Ces efforts faciliteront non pas l'âgisme, non pas une simple adaptation, mais une véritable intégration des personnes âgées dans la société canadienne. 


\section{Références}

Birren, J. (1987). The Best of All Stories. Psychology Today, May, 74-75.

Davidson, W. (1991). Metaphors of Health and Aging: Geriatrics as Metaphor. In G. Kenyon, J. Birren, \& J.J.F. Schroots (Eds.), Metaphors of Aging in Science and the Humanities (pp: 173-184). New York: Springer.

Kenyon, G., Birren, J., \& Schroots, J.J.F. (Eds.). (1991). Metaphors of Aging in Science and the Humanities. New York: Springer.

Lebel, P. (1991). Editorial: Does the Training in Geriatrics Affect a Doctor's Decision to Care for the Elderly? Canadian Journal on Aging, 10(3), 207-211.

Neugarten, B., \& Hagestad, G. (1976). Age and the Life Course. In R. Binstock \& E. Shanas (Eds.), Handbook of Aging and the Social Sciences (pp. 35-55). New York: Van Nostrand Reinhold.

Philibert, M. (1968). L'Échelle des Âges. Paris: Éditions du Seuil.

Gary M. Kenyon 